\title{
EFFECT OF PAIN COPING STRATEGIES V/S KINESTHESIA, BALANCE AND AGILITY EXERCISES IN CHRONIC KNEE OA: A CLINICAL TRIAL
}

\section{Rinkle Hotwani *, Shradha Shah, Soniya Keswani.}

MGM Medical college Aurangabad, Maharashtra, India.

\section{ABSTRACT}

Aim: This study is designed to determine better intervention strategies between pain coping strategies and kinesthesia, balance and agility exercises for chronic knee OA.

Study design: Experimental study, Randomized Clinical Trial.

Method: 40 participants both male and female with physician diagnosed chronic knee OA were assigned into two groups. The objective of the study was explained, and the subjects were screened based on the inclusion and exclusion criteria. Group A received kinesthesia, balance and agility exercises which include- Wedding march, Backward wedding march, Side stepping, tandem walk, toe walking, heel walking and then exercises progress into leaning forward, backward and sideways, rocker bottom balance board, carioca cross over maneuver and modified grapevine. Group B received Pain coping strategies involving 10 supervised sessions. Both the groups received treatment protocol of 6 weeks with sessions 3 times / week.

WOMAC scores were taken at baseline as well as after 6 week of treatment.

Results: Both the groups showed clinically and statistically significant improvement in WOMAC scores at the end of 6 -weeks of treatment session. i.e., $47.043 \pm 11.753$ to $5.815 \pm 11.079$ in group $A$ and $44.579 \pm 8.045$ to $27.849 \pm 5.216$ in group $B$.

Conclusion: A significant improvement were observed in both the groups; however the study has demonstrated that KBA exercises presented more beneficial effects in reduction of pain and improvement of functional status whereas pain coping strategies had beneficiary effects in cognitive and behavioral reactions to pain as they affect pain, functional capacity and psychological functioning.

KEY WORDS: Chronic knee OA, kinesthesia, pain coping.

Address for correspondence: Dr Rinkle Hotwani (MPT) Associate Professor, MGM Medical college Aurangabad, Maharashtra, India. E-Mail: drrinklemalani@gmail.com

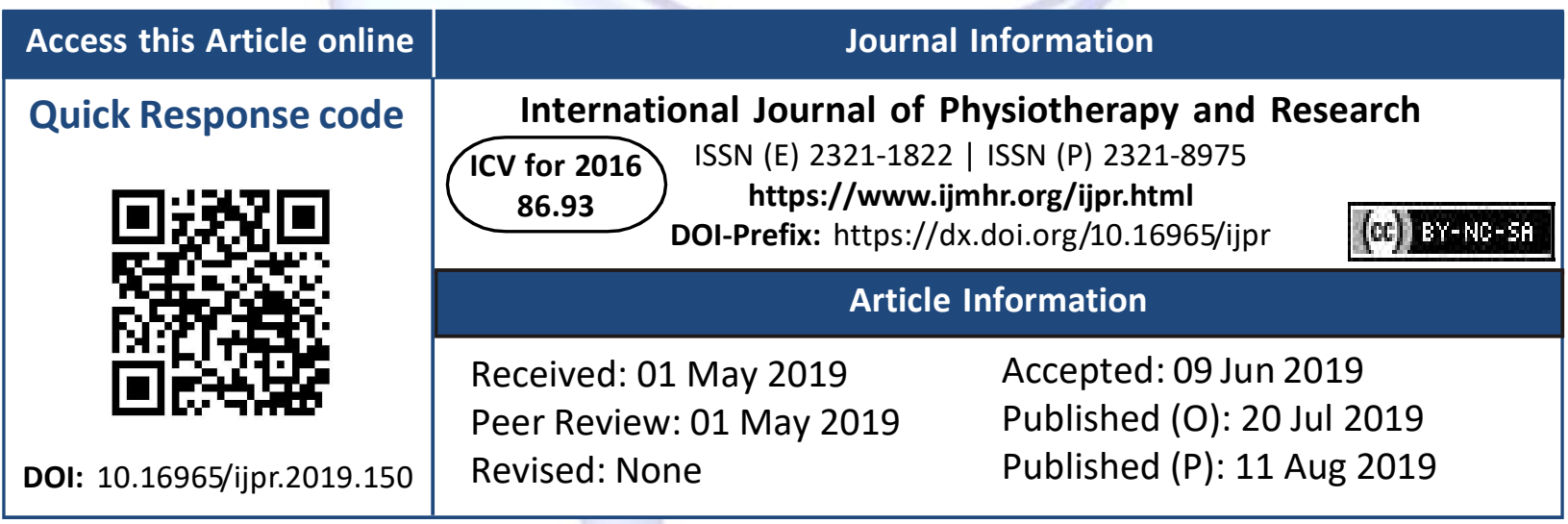

\section{INTRODUCTION}

The subcommittee of osteoarthritis of American college of Rheumatology diagnostic and therapeutic criteria committee defined osteoarthritis $(\mathrm{OA})$ as "A heterogeneous group of conditions that lead to joint symptoms and signs which are associated with defective integrity of articular cartilage, in addition to related changes in the underlying bone at the joint margins" [1].

Symptomatically the commonest joint affected is knee joint. The physical disability from knee OA prevents to perform activities of daily living and negatively affects quality of life [2]. Chronic knee pain is considered as prosaic, inevitable and unmanageable consequence of ageing. This leave unnoticed the socioeconomic burden caused by chronic knee pain as well overlooks the suffering, physical disability and psychological distress due to knee osteoarthritis [3]. 
It has been documented that proprioceptive acuity i.e. body segment positions and movements in space (kinesthesia), and sense of defense declines with age and as a result of OA. This lack of proprioceptive sense may contribute towards altered or reduced dynamic knee stability.

These Proprioceptive impairment can be effectively treated by giving Kinesthesia, balance and agility exercise which are mentioned to activate, challenge, and adapt the nervous system's proprioceptors. Decrease in proprioceptive deficit would thereby improve dynamic knee stability which improves the symptoms, slow down the disease progression and thereby improve activities of daily living [4].

Patients manage with chronic pain as an ongoing process which includes understanding stress, cognitive, behavioral and emotional coping response and subsequent reappraisals of stress.

Although the importance of decreasing maladaptive and encouraging adaptive coping response is emphasized by innovative treatment program for chronic pain [5]. Cognitive and behavioral reactions to pain are significant because they may affect pain, functional capacity, psychological functioning and may be amenable to change brought about by intervention [6].

As chronic knee OA is stressful condition and chronic pain is related with the emotional responses as stress, so coping strategies can be useful to decrease chronic pain by decreasing stress or emotional response.

Most patients with chronic diseases are unable to solve their persisting pain conditions by themselves though there are many ways to cope up with pain and there are different ways to regulate emotions associated with chronic diseases. Chronic pain patients find distance to negative emotions which are associated with pain, as well also tries to find strategies to adapt to a long lasting course of disease. Despite of long lasting courses of chronic pain, patients tends to find ways to maintain physical emotional and spiritual health [5].

Though there are several studies which show that osteoarthritis requires multifaceted treatment because of its clinical course so the present study designed to determine better intervention strategies among pain coping strategies and kinesthesia, balance and agility exercise for chronic knee OA.

\section{METHODOLOGY}

Data collected from MGM Physiotherapy Department and different Physiotherapy out Patient Department (OPD), Aurangabad. The study included male and female participants (Total 40 participants) with physician diagnosed chronic knee OA. The purpose of the study was explained, and the subjects screened based on the inclusion and exclusion criteria were allocated into 2 groups i.e., group $A$ (KBA group) and group $B$ (Pain coping group) and a written informed consent were taken from all the participants after their inclusion. Treatment sessions of 6 weeks i.e., 3 times / week were given and pre and post outcome measures were taken.

Inclusion criteria: Physician diagnosed primary chronic knee OA, unilateral or bilateral aged 45 years or over and of either gender.

Exclusion criteria: Any knee surgery or medical conditions precluding safe exercise.

\section{Procedure:}

GROUP A: (KBA group) - KBA exercises given to 20 participants. Before KBA exercises, a 5 minute walking warm up and after KBA exercise session, post-workout static stretching of calf, hamstrings and quadriceps muscle were given.

Participants were first shown static balance exercises on a stable surface and then progressed to dynamic balance exercises. After learning dynamic balance activities they progressed in the same manner. Dynamic balance includes the addition of small, rapid bouncing movements. To further perturb the balance and stimulate neuromuscular control mechanism addition of limb movements were given as a progression. Those participants who can achieve the target level were progress to another level of exercise. When a lower level continued to prove adequately challenging participant were not asked to progress through all levels of instability. Each balance exercise was given up to 3 sets for 30 sec.

KBA exercises includes- Wedding march, Backward wedding march, Side stepping, tandem walk, toe walking, heel walking and then exercises 


\begin{tabular}{|c|c|c|}
\hline PCST SESSION & CONTENT & HOME PRACTICE DOSAGE \\
\hline \multirow{2}{*}{$\begin{array}{l}\text { Session 1: progressive muscle } \\
\text { relaxation(PMR) }\end{array}$} & 1.Provide rational for PCST. & \multirow{2}{*}{ Practice of PMR twice/day } \\
\hline & 2.Training of participant & \\
\hline \multirow{2}{*}{ Session 2: mini practices } & 1.Review PMR & \multirow{2}{*}{ mini practices per day 10 or more } \\
\hline & 2.Mini practices taught to the participant & \\
\hline \multirow{2}{*}{ Session 3: activity-Rest-cycling } & 1.Review PMR and mini practices & \multirow{2}{*}{ Per week two sessions } \\
\hline & 2.Introduce activity rest cycling & \\
\hline \multirow{2}{*}{ Session 4: pleasant activity scheduling } & $\begin{array}{l}\text { 1.Set pleasant activity goals by describing } \\
\text { how this helps to control and decrease } \\
\text { pain with participant. }\end{array}$ & \multirow{2}{*}{3 pleasant activities/week } \\
\hline & $\begin{array}{l}\text { 2.Discuss how to use mini-practices and } \\
\text { activity-rest-cycling to attain pleasant } \\
\text { activity goals. }\end{array}$ & \\
\hline \multirow{2}{*}{$\begin{array}{l}\text { Session 5: identification of negative } \\
\text { thoughts, and calming self statements }\end{array}$} & 1. Follow thought process & \multirow{2}{*}{$\begin{array}{l}\text { Maintain record of daily } \\
\text { situations and thoughts. }\end{array}$} \\
\hline & $\begin{array}{l}\text { 2. Monitor negative thoughts and record } \\
\text { them. }\end{array}$ & \\
\hline $\begin{array}{l}\text { Session 6: test negative thoughts, } \\
\text { calming self statements }\end{array}$ & $\begin{array}{l}\text { 1. Invite negative thoughts and work with } \\
\text { participant to develop calm self-statements }\end{array}$ & $\begin{array}{l}\text { Develop alternative coping } \\
\text { thoughts daily. }\end{array}$ \\
\hline \multirow{2}{*}{$\begin{array}{l}\text { Session 7: problem solving, pleasant } \\
\text { imagery, distraction techniques }\end{array}$} & \multirow{2}{*}{$\begin{array}{l}\text { 1. Train participant in problem solving, } \\
\text { pleasant imagery and counting backwards }\end{array}$} & $\begin{array}{l}\text { One problem solving activity per } \\
\text { day }\end{array}$ \\
\hline & & 2 Pleasant imagery per day \\
\hline \multirow[t]{2}{*}{ Session 8: distraction techniques } & $\begin{array}{l}\text { 1. Use auditory stimulus and centre point } \\
\text { as distraction methods }\end{array}$ & \multirow{2}{*}{ Three Distraction techniques/week } \\
\hline & 2. Review of skills & \\
\hline \multirow{2}{*}{ Session 9: problem solving } & 1. Identifying problem situations & \multirow{2}{*}{$\begin{array}{l}\text { Maintain record of situation and } \\
\text { thoughts daily }\end{array}$} \\
\hline & 2. Developing coping plans & \\
\hline \multirow{3}{*}{ Session 10: coping skill maintenance } & 1. Review relapse prevention & \\
\hline & $\begin{array}{l}\text { 2. Identify foreshadowing of reduced } \\
\text { coping }\end{array}$ & \\
\hline & $\begin{array}{l}\text { 3. Devel op coping plans to address lapse in } \\
\text { coping }\end{array}$ & \\
\hline
\end{tabular}

progress into leaning forward, backward and sideways, rocker bottom balance board, carioca cross over maneuver and modified grapevine.

GROUP B: (Pain coping group)- Pain coping strategies were given to 20 participants for 6 weeks involving 10 supervised sessions (thrice weekly for 6 weeks).

\section{RESULT}

The study was conducted on 40 participants in which 24 females and 16 males were involved. It has been shown that women are more severely impacted by knee OA. Results indicate that there is significant improvement in WOMAC score at the end of the treatment in both groups i.e., KBA exercises and pain coping strategies. Both the treatment obtained reduction in WOMAC score i.e., $47.043 \pm 11.753$ to $25.815 \pm 11.079$ in group $A$ and $44.579 \pm 8.045$ to $27.849 \pm 5.216$ in group $B$. The result showed that patients of group $A$ had significant reduction in WOMAC score.
Table 1: Comparison of mean WOMAC score at pre and post in groups.

\begin{tabular}{|c|c|c|c|c|}
\hline & GROUPS & MEAN \pm SD & t value & p value \\
\hline $\begin{array}{c}\text { Pre } \\
\text { treatment }\end{array}$ & Group A & $47.043 \pm 11.753$ & \multirow{2}{*}{0.7737} & 0.4439 \\
\cline { 2 - 3 } $\begin{array}{c}\text { Post } \\
\text { treatment }\end{array}$ & Group B & $44.579 \pm 8.045$ & & \\
\cline { 2 - 3 } & Group B & $25.815 \pm 11.079$ & \multirow{2}{*}{0.7428} & 0.4622 \\
\hline
\end{tabular}

Table 2: Mean difference between pre and post WOMAC score in groups.

\begin{tabular}{|c|c|c|c|}
\hline & $\begin{array}{c}\text { Mean } \\
\text { difference }\end{array}$ & t value & $\mathbf{p}$ value \\
\hline Pre v/s post group A & 21.22 & 5.8 & 0.0001 \\
\hline Pre v/s post group B & 16.73 & 7.7 & 0.0001 \\
\hline
\end{tabular}

\section{mean difference}

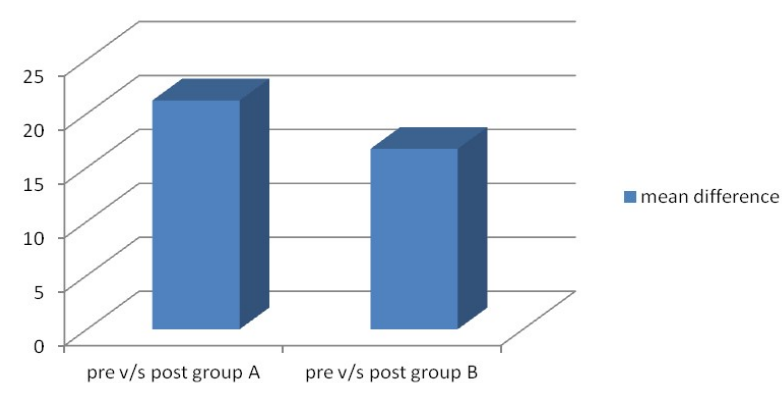




\section{DISCUSSION}

Results indicate that there is significant improvement in WOMAC score at the end of the treatment in both groups i.e., KBA exercises and pain coping strategies. The result showed that patients of group A had significant reduction in WOMAC score.

KBA exercises showed effect as propioception encompasses the sense of joint position as well joint motion. These senses originate from the stimulation of specialized nerve endings or mechanoreceptors in the joint capsule and ligaments. The receptors convert the mechanical energy of physical deformation into the electrical energy of a nerve action potential and this action potential propagates to the higher centre of motor control. Reflex contractions of muscles by stimulation of proprioceptors protects joint from mechanical insults. Conscious contractions are, in most cases too slow to prevent the injury, because their nerve paths are usually longer therefore slower. Proprioception involves multiple sensory systems like visual, vestibular, somatosensory, integumentary, joints, muscles, ligaments and tendons [7].

It has been documented that proprioceptive acuity i.e. body segment positions and movements in space (kinesthesia), and sense of defense declines with age and as a result of OA. This compensated proprioception affects dynamic knee stability. To minimize these proprioceptive deficits KBA exercises are designed which has potential to activate, challenge, and adapt the nervous system's proprioceptors. Furthermore dynamic knee stability increases with improved proprioception and showed better quality of life. However in context of development of knee OA joint instability and frontal plane joint laxity factors are considered as a probable causative factor with further erosion of articular cartilage among persons with knee OA. To slow down the disease's progression and improve symptomatically joint stability should be enhanced as well maintained. Sports agility training, primarily meant to improve the ability to more rapidly change limb or body positions. This makes KBA rehabilitation protocol differ from sport specific agility training as it efficiently changes body positioning, utilizing balance, coordination and speed in relation to the function required for normal daily activities, and hence KBA agility training is usually conducted at a walking pace [4].

Similar result was reported by Santosh Metgud and B.B. Putti (2015) that proprioceptive exercises led better outcome in proprioceptive function in patients with osteoarthritis as well knee replacement and these exercises can be added in rehabilitation protocol to improve daily activities and proprioception [1].

Sung-Bum et.al also concluded that a proprioceptive circuit exercise helps to improve knee joint muscle function and pain reduction in patients with knee OA. This study showed that these exercises can be effectively given to improve knee joint muscle function and decreased pain in the patients with knee osteoarthritis [8].

Matthew W. Rogers et.al proved that KBA is a promising stand alone intervention for improving function, pain and stiffness and it is an inexpensive easy to administer program of balance and agility exercises helps to improve functional capacity, decrease knee pain and instability and improves physical activity levels. These findings are complemented by other studies which states that KBA programs as an intervention are easy set of exercises to improve symptoms of knee OA and improve physical functioning more rapidly than strength training in the patients with chronic knee OA [4].

$O A$ is a multifactorial and complex chronic disease which contributes pain and disability. This supports the need for the biopsychosocial approach that addresses the psychological impairements that are commonly found. An individual's coping mechanism to deal with pain is of utmost importance [9]. Pain is considered as one of the most universal type of stress. Cognitive and behavioural reactions to pain are significant because they may feel pain, functional capacity, and psychological functioning and may be amenable to change brought about interventions [6]. A stressor can be defined as any stimulus that threatens normal homeostatic mechanisms. The neuroendocrine HPA stress axis has traditionally being considered to be a slower, back-up defence to stress. HPA axis is responsible for both cognitive appraisals of the stressful 
situations as well as the behavioural and endocrine adaptation to stress. However, continued and prolonged stress may disturb HPA axis to such an extent that the negative feedback mechanisms are disturbed: the adaptive responses to the HPA axis may then become maladaptive [10]. So far there are many ways to cope with pain, as chronic diseases needs to regulate emotions. To regulate such emotions and manage chronic pain innovative treatment programs emphasized by giving importance in decreasing maladaptive and encouraging adaptive coping responses [5].

A study done by Tamara J. Somers et.al (2012) supports the current study that behavioral interventions can be used as coping skills like lifestyle changes and weight loss strategies by training overweight and obese OA patients and they manage their weight as pain coping skills, which has their own additive benefits to cope up with chronic pain [11].

Many of individual limit their physical activity due to fear of increased joint damage, anxiety towards pain, avoiding social obligations, pain catastrophising and this behavior is considered as maladaptive coping mechanism for how they deal with pain and is of utmost importance as this can lead to reports of increased pain and functional decline. The research also suggests that pain catastrophising and self efficacy cognitive variables are important predictors of pain and function. Kim L Bennell et.al concluded that integrated exercise and pain coping skill training have been shown symptomatic relief and overall reduction in arthritis impact in knee $O A$ patient, they also provide a rational that an integrated pain coping skill training and exercise program is more potent than single intervention alone [9].

\section{CONCLUSION}

The study has demonstrated that kinesthesia, balance, agility exercises and pain coping strategies are effective to treat chronic knee OA. In addition the study concluded that KBA exercises presented more beneficial effects in improving pain and showing better functional status than pain coping strategies as KBA exercises helps in neuromuscular restoration with significant recovery in functional status of the patients and provide increase in strength of dynamic muscle activity.

Whereas pain coping strategies had beneficiary effects in cognitive and behavioral reactions to pain as they affect pain, functional capacity and psychological functioning.

Future recommendations: Present study used subjective outcome, more objective and functional outcomes can be used to establish the effectiveness of kinesthesia, balance and agility exercises and pain coping strategies in the treatment of chronic knee OA.

\section{Conflicts of interest: None}

\section{REFERENCES}

[1]. Santosh Metgud, B.B. Putti. Effect of proprioceptive exercises in osteoarthritic and replaced knees in Karnataka, India. Int J Physiotherapy Res 2015;3(6):1294-00.

[2]. Demirhan Duracoglu, Resa Aydin, Akin Baskent, Ajda Celik: effects of kinesthesia and balance excercises in knee osteoarthritis, JCR: journal of rheumatology, 2005;11(6).

[3]. M.V. Hurley, N.E. Walsh, H.L Mitchell, T.J. Pimm et.al: clinical effectiveness of a rehabilitation program integrating exercise, self management, and active coping strategies for chronic knee pain: A cluster randomized trial. arthritis and rheumatism, 2007;57(7):1211-1219.

[4]. Matthew W. Rogers, Nauris Tamulevicius, Marius F. Coetsee, Beth F. Curry, and Stuart J. Semple: knee osteoarthritis and the efficacy of kinesthesia, balance and agility exercise training: a pilot study in university of Zululand, south Africa; Barry university,Miami, USA; Morton plant Mease Health care, USA.

[5]. Arndt Bussing, Thomas Ostermann, Edmund AM Neugebauer, and Peter Heusser: adaptive coping strategies in patients with chronic pain conditions and their interpretation of disease.BMC public health 2010;10:507.

[6]. Kraaimaat FW, Evers AW. Pain-coping strategies in chronic pain patients: psychometric characteristics of the pain-coping inventory $(\mathrm{PCI})$. International journal of behavioral medicine. 2003 Dec 1;10(4):343-63.

[7]. Ashish kumar: joint proprioception in normal and osteoarthritic knees, in national institute for orthopedically handicapped, Bon Hoogly, BT road, Kolkata, India. J yoga physical therapy 2012;2:4.

[8]. Sung-Bum Ju, Gi Duck Park, Sang-Soo Kim: Effects of proprioceptive circuit exercise on knee joint pain and muscle function in patients with knee OA, journalof physical therapy science 21 aug 2015. 
[9]. Kim L Bennell etal : A Physiotherapist- delivered integrated exercise and pain coping skills training intervention for individuals with knee OA: a randomised controlled trial protocol, BMC Musculoskeletal disorders 2012;13:129.

[10]. G Blackburn- Munro and R.E. Blackburn Munro: chronic pain, chronic stress and depression: coincidence or consequence? In Adenburg, UK, journal of neuroendocrinology, 2001;13:1009-1023
[11]. Tamara J. Somers et.al. Pain coping skills training and lifestyle behavioural weight management in patients with knee osteoarthritis: A randomized controlled study. 2012 Jun;153(6):1199-1209.

How to cite this article:

Rinkle Hotwani, Shradha Shah, Soniya Keswani. EFFECT OF PAIN COPING STRATEGIES V/S KINESTHESIA, BALANCE AND AGILITY EXERCISES IN CHRONIC KNEE OA: A CLINICAL TRIAL. Int J Physiother Res 2019;7(4):3157-3162. DOI: 10.16965/ijpr.2019.150 